Proceedings of the ASME 2021

International Design Engineering Technical Conferences and

Computers and Information in Engineering Conference

IDETC/CIE2021

August 17-20, 2021, Virtual, Online

\title{
DETC2021-69781
}

\section{INTEGRATING HEDONIC QUALITY FOR USER EXPERIENCE MODELLING}

\author{
Yanzhang Tong ${ }^{1 *}$, Yan Liang ${ }^{1}$, Ying Liu ${ }^{1}$, Yulia Hicks $^{2}$, Irena Spasic ${ }^{3}$ \\ ${ }^{1}$ High-Value Manufacturing Research Group, School of Engineering, Cardiff University, Cardiff, CF24 \\ 3AA, UK \\ 2 Medical Engineering Research Group, School of Engineering, Cardiff University, Cardiff, CF24 3AA, UK \\ ${ }^{3}$ School of Computer Science and Informatics, Cardiff University, Queen's Buildings, Cardiff CF24 3AA, \\ UK. \\ * Corresponding author. Tel.: 07399235522. Email address: TongY4@cardiff.ac.uk
}

\begin{abstract}
Research on user experience (UX) has attracted much attention from designers. Additionally, hedonic quality can help designers understand user interaction (such as attractive, original and innovative) when they experience a product. Realising the user's interaction state is a significant step for designers to optimise product design and service. Previous UX modelling lacks exploration in user interaction state. Also, the lack of user interaction state factor will reduce the accuracy of the UX modelling. In this paper, we explore the interaction value of online customer review and introduce a new approach to integrating hedonic quality for UX modelling. Firstly, extracting word list from online customer review; Secondly, hedonic quality words are extracted from the word list and added as a hedonic quality part to UX modelling; Thirdly, we compared the analysis result with our previous study for the conclusion. This research combines hedonic quality with UX modelling to enrich modelling in the field of UX for the first time. The proposed data collection method is superior to the traditional collection methods in hedonic quality studies. Extracting hedonic quality factors from online customer reviews can in-depth provide reflections for designers to improve their product design. Furthermore, it also explored the valuable relationship between $U X$ and online customer reviews to provide proactive thinking in user strategy and design activities.
\end{abstract}

Keywords: Design informatics, User experience, Hedonic quality, Opinion analysis, Product design

\section{INTRODUCTION}

In the early design stages, an analysis to identify product opportunities and attractive conceptual ideas is vital for the product design because the customers prefer to interact with attractive products. Designers and companies increasingly believe that user experience (UX) can provide essential information for product design [1, 2]. UX is defined as an objective feeling user get when they interact with an artefact, a particular product, system, service or object $[3,4]$. More specifically, UX focuses on users' feelings stemming from the pragmatic and hedonic aspects. According to the International Organization for Standardization (ISO), several significant factors impact UX, such as user's internal and physical states, product properties and the context of use [5], where the hedonic aspect is a key attribute to affect the product's acceptance [6]. Hence, hedonic quality is considered significant for UX measurement.

With the fast development of various social media platforms, a large number of researchers consider user-generated content (UGC) as a significant and valuable resource for researches and commercial uses, such as sentiment analysis [7], product purchase suggestion [8] and customer concerns summarisation [9]. Therefore, information on UX can be obtained from online customer reviews [10]. However, online 
reviews normally contain unstructured forms of natural language that is not straightforward for researchers or designers to analyse on a large scale. Simultaneously, sentiment analysis or online opinion analysis is usually used to dig out customer's sentiments which limits uncovering the information of UX or hedonic quality from UGC.

In light of these challenges and the rising need for UX analysis, it is desirable to automate the management of the knowledge of hedonic quality. Based on the previous work of UX modelling [11], this paper introduces a new approach to integrate hedonic quality into UX modelling. Firstly, this paper briefly introduces our previous research efforts [11] investigating the hedonic quality factors on user experiences and then propose a new approach by integrating hedonic quality for UX modelling. Next, based on Amazon online customer reviews, a case study was carried out for validating the effectiveness of the proposed model. Finally, the experimental results are presented and discussed, followed by concluding remarks that reflect the study and possible future explorations.

\section{LITERATURE REVIEW}

\subsection{Hedonic quality}

Some critical UX researchers believe that user experience is shaped by a combination of pragmatic ("instrumental product"/"task-oriented"/"ergonomic") qualities and hedonic ("non-instrumental"/"non-task-oriented") qualities of a system [12-14]. It is necessary to clarify what is pragmatic quality and hedonic quality, and it is shown in TABLE 1 . Pragmatic quality is defined as the realised usefulness, efficiency, and ease of use. It is also referred to as utility and usability aspects. The hedonic qualities take into account the "joy of use" and emphasise stimulation, identification and evocation generated by the use of a system or a product. It is the aggregate of pragmatic quality where hedonic qualities can lead to positive or negative emotions and consequently guides the product's acceptance [15]. Pragmatic product attributes and hedonic attributes are the main contributor to the acceptance of the product and help users achieve hedonic goals [6]. Minge illustrated that overshadowing effects of hedonic aspects are more important than pragmatic

\begin{tabular}{|c|c|c|}
\hline & Definition & Relation \\
\hline $\begin{array}{c}\text { Pragmatic } \\
\text { quality }\end{array}$ & $\begin{array}{l}\text { pragmatic qualities refer to } \\
\text { the perceived usefulness, } \\
\text { efficiency, and ease of use } \\
\text { (so-called utility and } \\
\text { usability aspects }\end{array}$ & $\begin{array}{l}\text { It is the } \\
\text { combination of } \\
\text { pragmatic and } \\
\text { hedonic qualities } \\
\text { that leads to }\end{array}$ \\
\hline $\begin{array}{c}\text { Hedonic } \\
\text { quality }\end{array}$ & $\begin{array}{l}\text { hedonic qualities take into } \\
\text { account the "joy of use" and } \\
\text { emphasise stimulation, } \\
\text { identification and evocation } \\
\text { generated by the use of a } \\
\text { system or a product }\end{array}$ & $\begin{array}{l}\text { negative } \\
\text { emotions and } \\
\text { consequently } \\
\text { guides the } \\
\text { acceptance of } \\
\text { the product. }\end{array}$ \\
\hline
\end{tabular}

TABLE 1. PRAGMATIC QUALITY AND HEDONIC QUALITY aspects [16]. However, Van der Heijden discovered that the different purposes of the system impact UX, and this means usefulness loses its power in hedonic systems, where it is the perceived enjoyment that becomes predictive of the user's acceptance [17]. This suggests that the emotional, aesthetic, and cognitive of products may be the key factors to indicate positive or negative user experience [15].

In order to distinguish between pragmatic quality and hedonic quality, and also to focus on beauty and goodness, some researchers developed different standardised questionnaires to measure hedonic quality, e.g., AttrakDiff [18, 19], the user experience questionnaire (UEQ) [20], and meCUE2.0 [21]. Increasing the speed and breadth of collecting hedonic quality information is an important step for future research.

\subsection{UX modelling and analysis}

Different researchers illustrate and define UX from different perspectives to align it with their research and application purposes. Some researchers believe that UX is holistic, while others suggest that summative and evaluative constructs form complexity experience, for example, emotions and usability [1]. In addition, several researchers highlight the effect of UX factors, such as usage context, user's state, and product properties [12].

When the researcher focuses on specific constructs of UX and their correlations, they can obtain and analyse user's ratings of these constructs for UX investigation. Some researchers analyse users' psychophysiological responses when interacting with products and services by conventional approaches, such as the user's emotion changes by electromyography (EMG). Additionally, the system's comment is collected by self-report [22]. However, it is difficult to analyse some effects on UX by psychometric instruments, such as product features, properties and the context of use [11].

Researchers also adopt other methods to analyse user's subjective responses to products, such as surveys, questionnaires, self-report [11]. Hassenzahl and his research partners tried to use pre-defined questionnaires to understand what constitutes "pleasant experiences" when interacting with the product, such as pragmatic quality, hedonic quality, and need fulfilment [23]. UX Curve method is able to help user retrospectively record the reasons for experience change at different times. For instance, several specific UX viewpoints (such as attractiveness, utility, ease of use and general UX ) transform user-generated data into different UX curves [24]. On the other hand, some research focused on the factors that influence a user's experience with a product and the cause-andeffect relations between factors [11].

In summary, previous studies mainly focus on conventional methods (e.g., surveys, questionnaire, and interview) to measure UX. However, these methods are time-consuming, making it hard to identify all contexts and features that significantly impact UX. To tackle this, some researchers explored new approaches to take advantage of online customer reviews for UX studies [11]. This paper focuses on the impact factors of UX and enrich 
previous UX modelling [11]. Many hedonic quality factors can be uncovered from online customer reviews.

\subsection{Mining of online customer reviews}

With the increase of social media platforms, people have generated numerous user-centric contents where online customer reviews include rich information, for instance, user experience, customer complaints, purchase and so on [25]. Compared with the traditional rating survey, this information describes the customer experience in a more detailed way so that it can more accurately reflect the views of the customer. In addition, an online product review can bridge the needs of customers and designers [26].

In the product design research area, online customer reviews can provide valuable and crucial information in terms of customer preferences, user needs, and design requirements. However, a lack of research focuses on plenty of online reviews for UX modelling and management. Meanwhile, a lack of studies explored UX modelling and online customer review in the online product online review research field [11]. To research online customer review, in this study, we explore a new approach to investigating hedonic quality for UX modelling.

On the other hand, researches on UGC opinion mining focus on identifications of product features and sentiments related to products or product features [27]. Semantic orientation (such as positive, negative and neutral) of opinions was identified, and Ding, Liu, and Zhang found product entities. Linguistic features

(e.g., pronouns, language conventions and comparative sentences) were assigned by them [28]. In 2010, Wang et al. acquired hotel user's opinions on product and service by the topical aspects, such as room, location and service. Furthermore, they provided a probabilistic model to extract the topical keywords as topical aspects and weights on aspects [29]. Meanwhile, Chen et al. predicted the overall sentiment from past tasks though retaining the knowledge and used the sentiment to aid future learning [7].

Some research tried to allocate product reviews into different groups at the product feature level, such as positive, negative and neutral classes. Popescu and Etzioni used a relaxation labelling method to find the semantic orientation of words for opinion phrases and sentiment polarity identified from reviews [30]. Also, Yang et al. introduced a method for UX modelling based on machine learning to extract many users' online customer reviews and automatically identify UX centric contents to obtain customer UX. Moreover, this method is capable of providing UX advice for optimisation of product design, and it is suitable for further UX exploration [11].

In light of the lack of research in harnessing the merits of UX and its immense value to product design, this paper focuses on integrating hedonic quality for UX modelling. The proposed approach can fill the research gap in UX to further UX research exploration and optimisation of product and service experience.

\section{APPROACH}

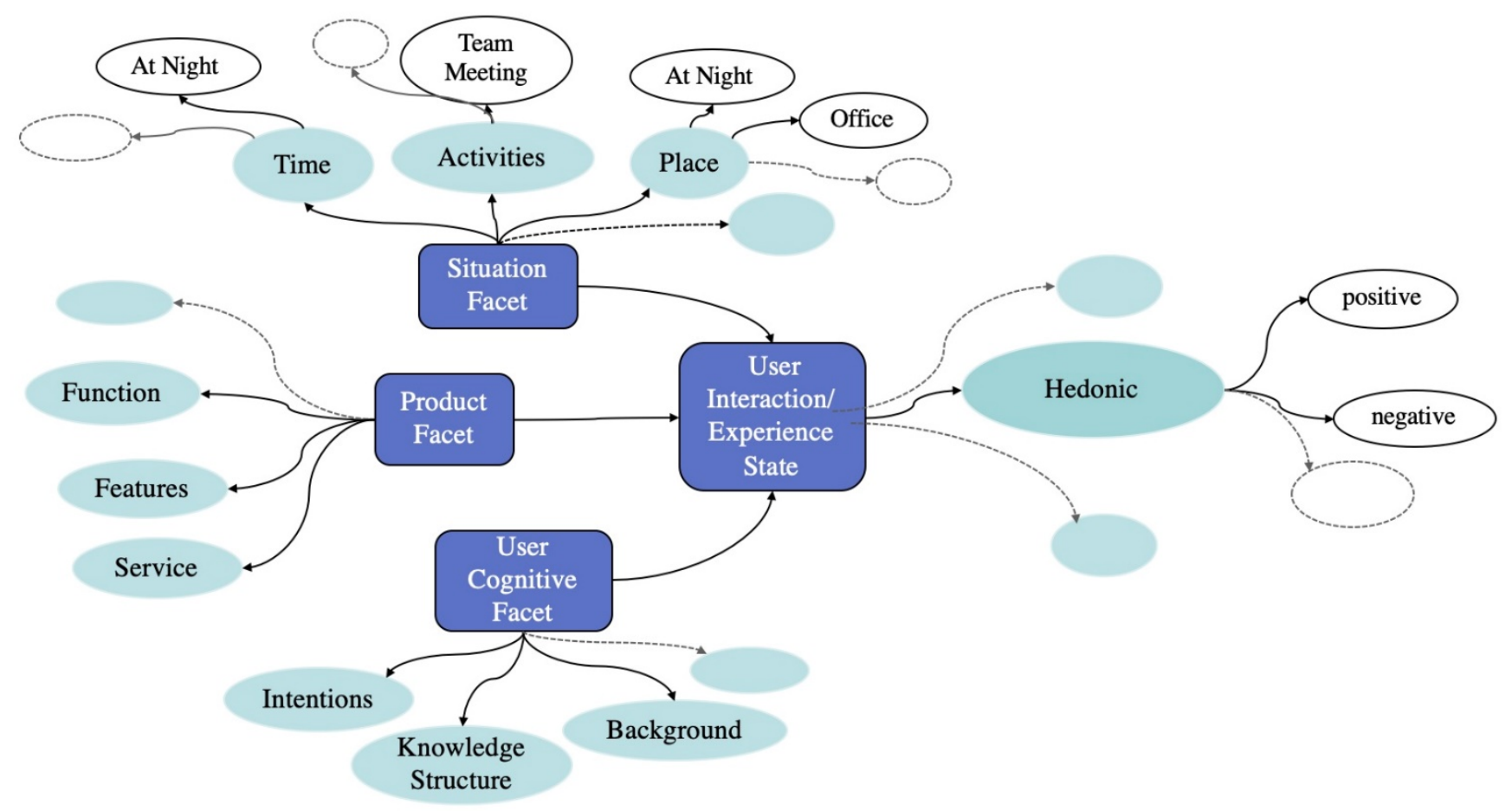

FIGURE 1. A FACETED MODEL FOR USER EXPERIENCE. 
The proposed UX model addresses a fundamental issue. Many UX models use traditional surveys, questionnaires, and interviews to acquire customer rating and experience input from product and service. Moreover, these traditional methods incur time cost to find potential users to answer questions. Customer concerns are overlooked if the designer suggests the questions. This type of UX data is often unstructured textual data documented by self-report or narrative [11]. The UX measuring process's complexity will be influenced by the number of UX factors, the specific UX factors involved, and their explicit or implicit descriptions. So if the UX model includes multiple relations or factors, it will need more human resources to process UX information.

UX modelling for measure and structure, user's affective and cognitive perspectives is UX definition, such as hedonic quality, beauty, and goodness. Some researchers define some critical words about hedonic quality by questionnaires, such as AttrakDiff [18, 19], the user experience questionnaire (UEQ)[20], and meCUE2.0 [21]. Furthermore, these hedonic quality keywords can help us to in-depth measure UX. On the other hand, they include some other important factors, which can have a significant influence on UX, such as product functionality, product service, and interaction situation [12].

Therefore, we propose a model of many aspects of UX to support UX information analysis by the customer narrative data including product properties, contextual factors, user cognitive facet and user's state, while the user's state is mainly about the user's sentiment state, i.e., positive, negative, or neutral about the interactions with a product. In this paper, to better understand, explore and analyse user's feelings about interactions with a product or service, we further study the user's state from a different perspective. We integrate hedonic quality into our previous UX modelling to extend the study of user's feelings in terms of hedonic aspects of the product. Technically, we explore a computational approach to extract and estimate users' feelings in hedonic quality aspects.

The hedonic-extended UX model is described as follows. The product facet $F$ of UX refers to the product characteristics involved in UX. The situation facet $S$ describes the contextual factors when using a product. The users' interaction state $Y$ is represented by the broader range of user experience state about product facet under certain situations, such as sentiment, hedonic quality, etc. UX can be denoted as Eq. (1). It indicates that interaction state $Y$ is collectively influenced by product feature $F$, situation feature $S$ and user cognitive facet $U$.

$U X: \forall \exists(F, S) \cup U \Rightarrow Y$

Based on the hedonic-extended UX model, the proposed

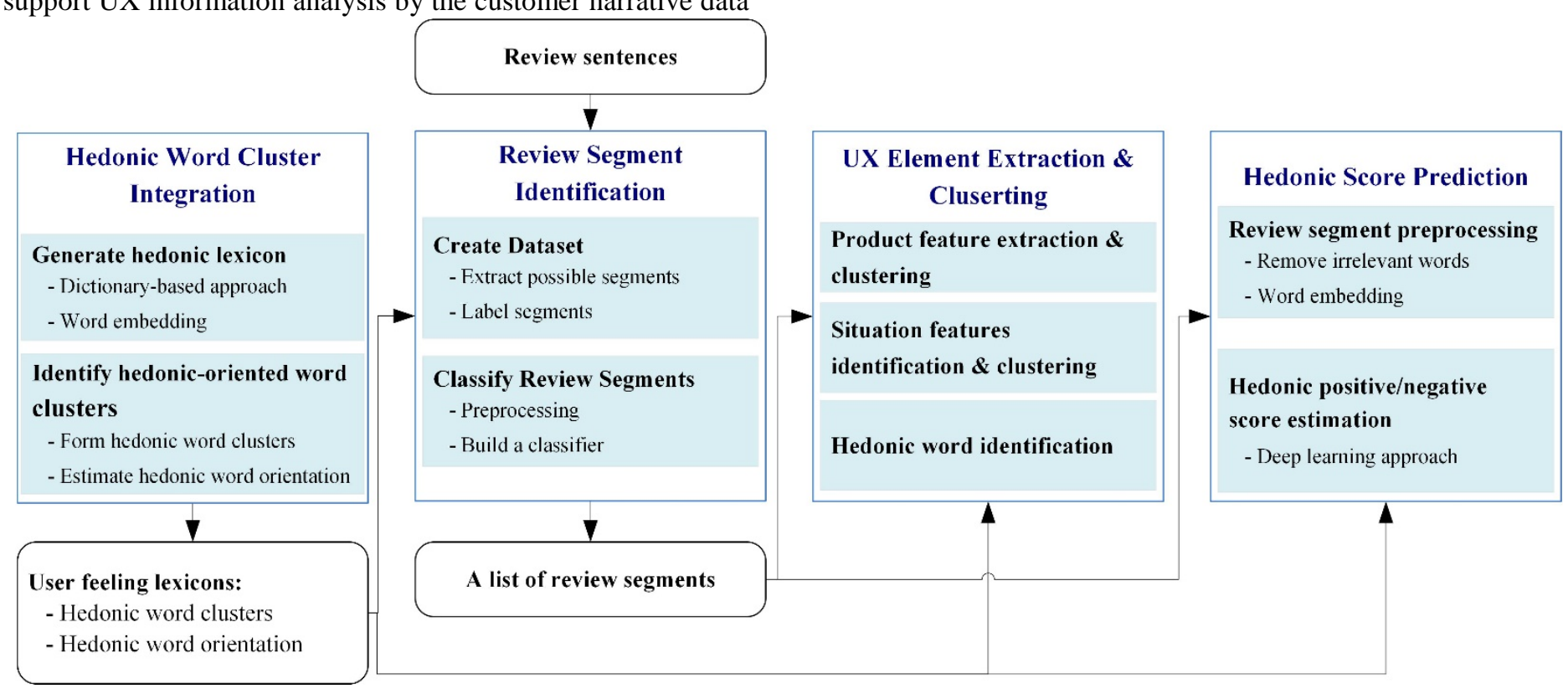

\section{FIGURE 2. THE APPROACH TO DISCOVER HEDONIC ASPECTS OF UX FROM ONLINE CUSTOMER REVIEWS}

(e.g., online product review). In short, this model will explain the user interaction state, which combines the product and service interaction process in different aspects (including product facet, cognitive facets and situation facet), which is shown in FIGURE 1.

In our previous study, we have explored UX modelling from massive online user reviews by using text mining techniques [11]. We covered four main categories of influences on UX, methodology consists of four steps to automatically measure hedonic aspects of UX from a large number of online customer reviews, as shown in FIGURE 2. Each step of the methodology is discussed in the following subsections.

\subsection{Hedonic word cluster integration}

Different users may use different hedonic words to express their feelings about the product. To analyse users' 
interaction/experience state from hedonic aspects, the first stage is to build up hedonic word clusters to describe the hedonic aspects that the UX researchers are concerned with. This stage starts with identifying core hedonic words based on the domain knowledge and then extends the core words using natural language processing (NLP) techniques.

On the one hand, we analyse the UQE designed by the UX researchers and then summarise the hedonic aspects that are often studied. The frequent hedonic words used in the questionnaires are extracted as core hedonic words. Each core word is tagged with a positive/negative label and a cluster id. Then by using lexicons, e.g., WordNet and google dictionary, the synonyms of each hedonic core word are extracted and added to the cluster where the core word belongs to. In addition, word embedding techniques are used to find similar words to the core hedonic words. The pre-trained word vectors built from massive online data, e.g., word2vec-google-news-300, are used to

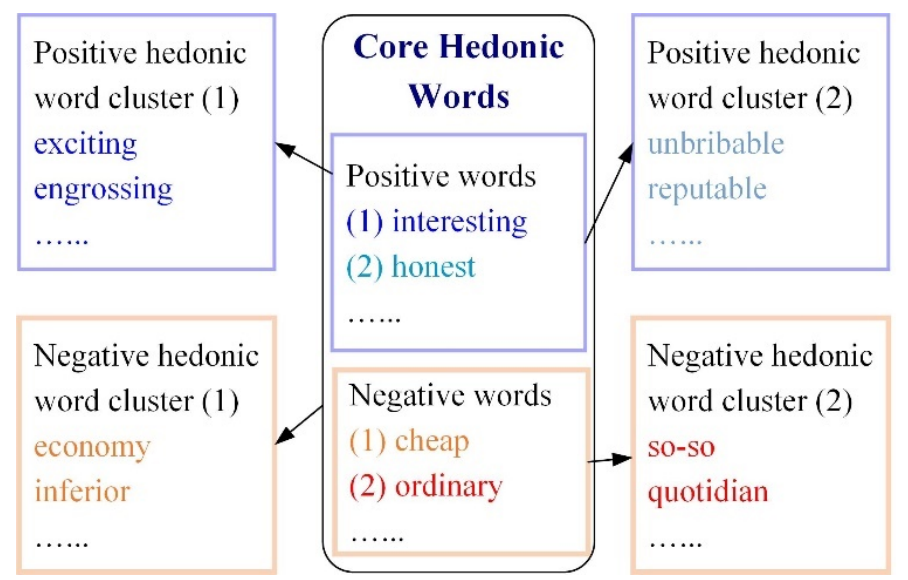

\section{FIGURE 3. HEDONIC WORD CLUSTERS}

calculate word similarity, and then top $k$ words are included in the corresponding cluster. We also trained the word vectors using the product review data by word embedding techniques to get similar words.

On the other hand, opinion words used to express a subjective opinion are extracted by using the approach in our previous study [11]. For each opinion word, if its synonyms appear in one or more hedonic word clusters, the opinion word is included in the cluster with the largest number of shared words. The remaining opinion words that are not assigned to any hedonic word cluster are grouped using the $x$-means clustering approach to form new word clusters. By combining word in positive and negative hedonic clusters, respectively, we can obtain two hedonic word lists, i.e., positive and negative hedonic word lists.

\subsection{Review segment identification}

At this stage, we aim to identify segments from each review sentience so that each segment contains information of hedonic aspects. From the product reviews, we notice that users sometimes write long sentences to describe their feelings, which probably contain several segments containing different hedonic

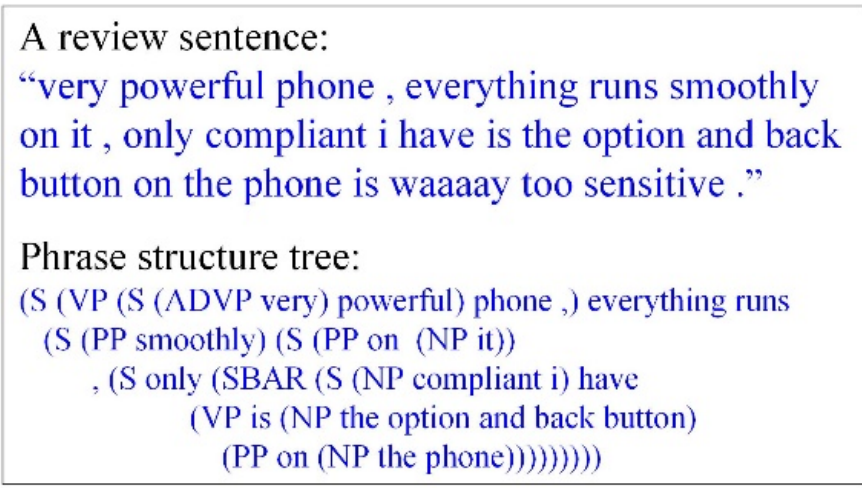

\section{FIGURE 4. CONSTITUENT TREE OF A REVIEW SENTENCE}

words, as shown in FIGURE 4. Therefore, to better analyse the hedonic aspects, we focus on segments with hedonic words rather than the sentence level.

The review opinion-segments are usually short subsentences with hedonic words. The syntactic analysis to understand the phrase structure [31] and constituent parts are useful to detect the boundary of the opinion segments. In our approach, the first step is to pre-processing review sentences. Each product review is parsed into a set of sentences, using some normal punctuations, e.g., full stops and question marks. Next, the sentences are parsed into phrase structure tree with constituent labels. The words in each sentence are also labelled with part-of-speech (POS) tags [32].

By using the phrase structure tree, a depth-first bottom-up approach is designed to form possible segments. Starting from the word at the bottom of the tree, the word with a shared parent node is combined until a node labelled with S (i.e., sentence) is reached. These words form a candidate segment. If this segment contains any hedonic word, then it is considered as a valid review segment, and then the process will traverse from another word at the bottom of the tree. Otherwise, this segment is further expanded by traversing up the constituent tree until another $\mathrm{S}$ label is meet. This process continues when all the nodes in the phrase structure tree are traversed. If a sentence does not contain any hedonic word, then it is filtered out.

It is noticed that even a review segment carrying a hedonic word may not related to the customer's feelings about the product, e.g., "good lucky people”. So, we build a classifier to filter the sentences that do not contain hedonic information about the product. The process first creates labels for segments, i.e., whether or not the segments contain hedonic aspects of the product, based on grammatical rules, i.e., whether there is a dependency relationship between product words and the hedonic words appearing in the segment. Therefore, the labelled sentences are checked manually and become a training set. Then using the training set, a classifier is trained to classify the unlabeled segments.

\subsection{UX element extraction and clustering}

Once the review segments have been obtained, the next step is to extract product features, e.g., "app" and "battery life" and 
situation features, like " reading their email" and " for business and personal uses". We adopted our previous approach to extract these UX elements [11].

In addition, to better analyse what product features or usage context are related to positive/negative hedonic aspects, UX data of these two feature types are integrated into clusters, respectively, for further analysis. The situation features are various in length and types of phrase structure. The cluster number is also difficult to estimate. Therefore, using word vectors, the x-means clustering is adopted [33]. Starting from one cluster, it can subdivide the cluster repeatedly until a statistical criterion such as the Bayesian information criterion (BIC) is reached.

It is noticed that even a review segment carrying a hedonic word may not be related to the customer's feelings about the product, e.g., "good luck people". So, we build a classifier to filter the sentences that do not contain hedonic information about the product. The process first creates labels for segments, i.e., whether or not the segments contain hedonic aspects of the product, based on grammatical rules, i.e., whether there is a dependency relationship between product words and the hedonic words appearing in the segment. Therefore, the labelled sentences are checked manually for their inclusion of a training set. Then using the training set, a classifier is trained to classify the unlabeled segments.

\subsection{Hedonic score prediction}

This stage aims to predict a hedonic score for each review segment to quantify a user's feeling of hedonic aspects. Hedonic scoring will help UX modelling to summarise the interaction state. Using review segments extracted as inputs, the preprocessing is about to remove stop words from the review segments, and the remaining words in each segment are represented by word embedding vectors. As for the hedonic score, it is predicted by applying machine learning approaches for sentiment analysis. In the training process, both word vectors and grammatical structures are used. The method outputs the two sentiment scores with respect to positive and negative sentiments in the range $[0,1]$. In this paper, the largest number is selected as the hedonic score. As for the negative sentiment, its negative value is used as the score. Therefore, the range of the hedonic score is $[-1,1]$.

\section{CASE STUDY}

In this section, a series of experiments are carried out where the results are compared and discussed to demonstrate the effectiveness of the proposed method. An illustration of UX modelling based on online customer reviews is presented, including 1) the hedonic data discovery; 2) UX knowledge base examples. To demonstrate the feasibility of the proposed approach, research data was crawled from Amazon.com, where the reviews targeted two types of smartphones (i.e., "Samsung Galaxy siii" and "Samsung Galaxy sii").

\subsection{Experiment design}

In this work, the information is automatically collected from UGC (i.e., online customer reviews) as it is much more timesaving and can provide a wider range of research samples for UX analysis. For hedonic quality analysis, the proposed method provides an automatic way to expand the scope of the research, where the expanded words of hedonic quality are used to improve the identification range of customer reviews. Moreover, these expended words are used to filter hedonic quality words in an automatic way to improve efficiency.

\subsection{Data collection}

In 1145 product reviews, more than 10,000 review sentences are included in this dataset. Each review contains 8.7 sentences or 155 words on average, and each sentence has approximately 17.7 words. To pre-process these data sets, we randomly selected 1130 reviews from these online customer reviews. Based on the methodology for building corpus [34], a co-author manually read all reviews in parallel but independently. It was found that the reviews were categorised as positive or negative (i.e., "interesting" and "boring") concerning product features, situation features and user hedonic quality opinions. Once an individual annotation has been completed, the annotated data were compared. If there was a disagreement, it required a discussion to reach a consensus. Finally, 1130 review sentences were tagged with hedonic quality polarity. In the experiments, standard text pre-processing was performed for each sentence, including lowercasing words, removing stop words [35] and stemming each word [36].

FIGURE 5 shows the experimental results of the proposed approach. According to the traditional hedonic quality questionnaire, some words related to the hedonic quality were summarised. Using the WordNet and Google dictionary, the hedonic quality core words were expanded by adding their synonyms and finally formed the positive and negative word sets. Meanwhile, relevant examples in the customer reviews were able to be found.

\subsection{Product feature clustering results}

This section demonstrates the effectiveness of the classifier in the proposed methodology. This subsection further analyses the obtained clusters of product features. Once the bigrams have been clustered into product feature clusters, the customer review sentences can be assigned to the clusters based on the product features that are contained in the sentence. FIGURE 6 and FIGURE 7 show the proportions of product feature for both phone data sets (i.e., positive and negative). Each fraction in FIGURE 6 and FIGURE 7 labelled by the most frequent product features in the cluster. It can be seen that product features were identified from the review datasets.

FIGURE 6 and FIGURE 7 show that customers have different opinions about product features. FIGURE 6 describes the top 10 product features with negative scores. It shows that customers pay more attention to the battery, screen and camera. About $35 \%$ of the customer reviews are not satisfied with the 


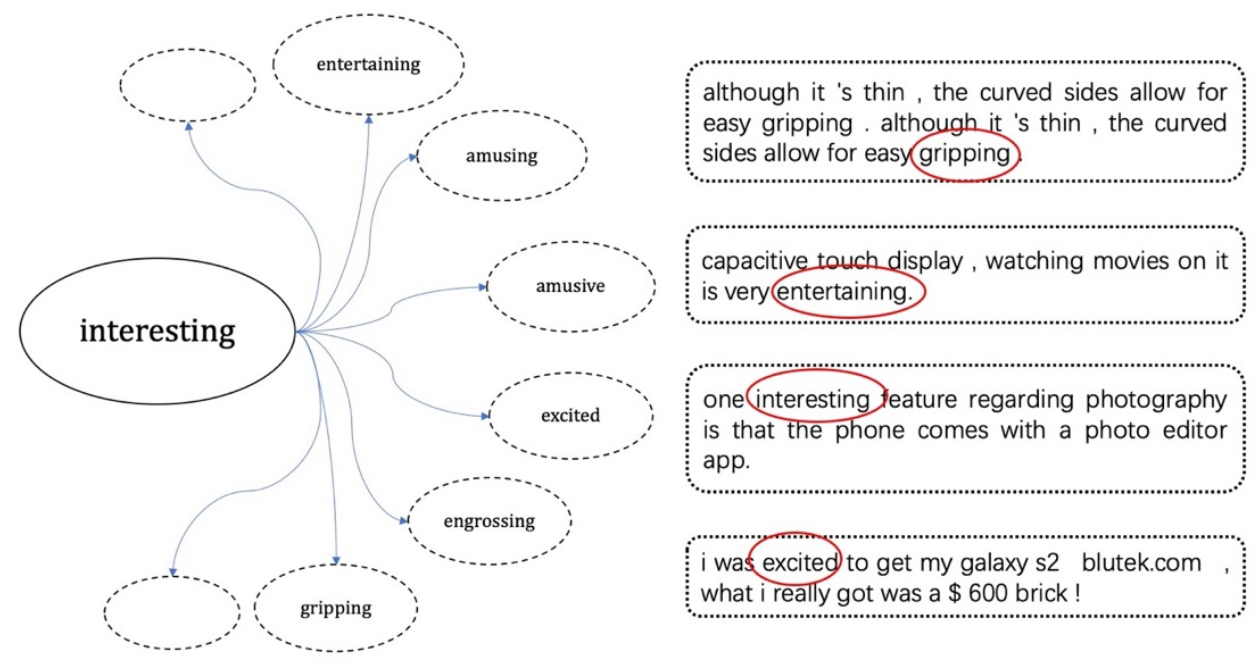

FIGURE 5. EXAMPLE OF HEDNOIC SYNONYMS AND RELATED REVIEW

battery of the Samsung phone, which leads to negative comments. About $17 \%$ of customer reviews indicate the screen of the Samsung Galaxy sii is considered unsatisfactory. It can be seen from these results that product features are significant to customers for giving positive/ negative online reviews when interacting with products. Besides, FIGURE 7 shows the top 9 positive factors of product features, such as screen, camera, quality and so on. About $29 \%$ of customer reviews are in favour of Samsung screens. Samsung's camera is considered satisfactory by $21 \%$ of customers. So, the ranking of positive reviews and negative reviews of product features indicate the product features that customers most concern about. Designers can improve the design of the products based on negative feedback from online reviews. On the contrary, the positive reviews about product features imply that designers should keep the advantages of the products.

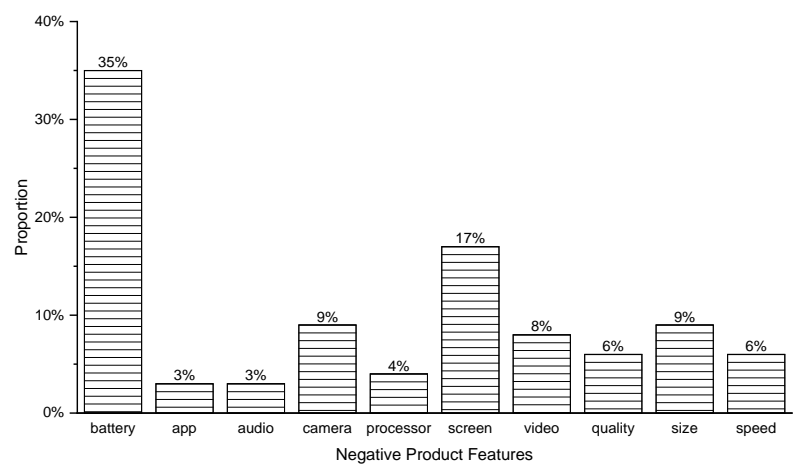

FIGURE 6. NEGATIVE REVIEW OF PRODUCT FEATURE

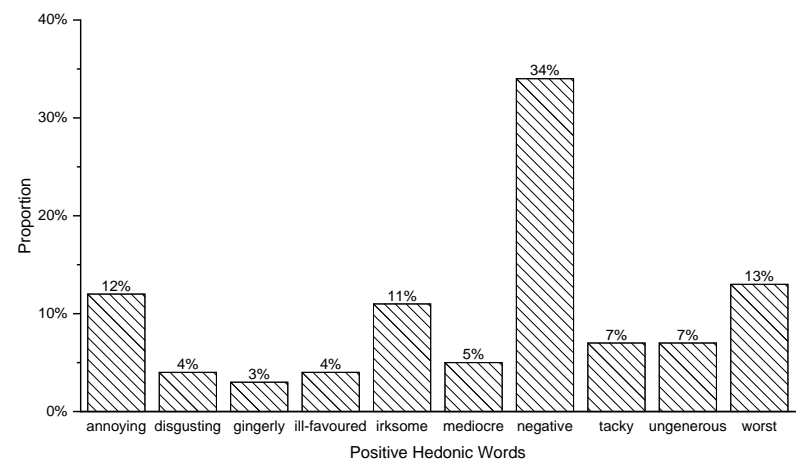

FIGURE 7. POSITIVE REVIEW OF PRODUCT FEATUR

\subsection{Hedonic quality clustering result form product features}

Based on the product feature clusters, further investigation on the hedonic quality of different product features was conducted. For example, the number of the product feature (e.g., "screen", "battery") that appears in review segments with a positive or negative hedonic score was counted.

FIGURE 8 shows the top 10 hedonic quality words extracted from online reviews on the product feature "screen". About $52 \%$ of review sentences indicate that the screen is interesting, while about $14 \%$ of reviews shows the screen is dramatic. Within $10 \%$ of review segments, "grateful" and "glossy" are the main reasons why customers have positive feedback to screen. Other positive feedback about the screen includes "chic", "auspicious", "fab", and "loveable". According to the results, it is clear to see that a large amount of hedonic 
quality information is covered in the sentences for evaluating product features. In addition, designers can dig out the reason why customers make positive feedback on a specific product feature through hedonic quality words analysis. This method provides a promising way for helping designers understand product UX in-depth to improve product design.

FIGURE 9 illustrate the top 8 negative hedonic quality words related to the battery. About $34 \%$ of review sentences

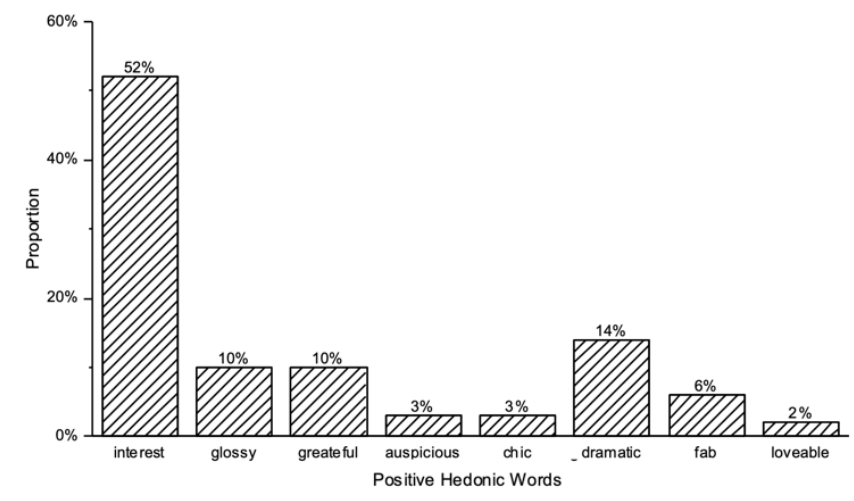

FIGURE 8. POSITIVE HEDONIC WORDS IN “SCREEN”

indicate the battery is negative, while about $13 \%$ of review sentences indicate that the battery is worst. The battery is considered annoying by about $12 \%$ of customer reviews. Other negative hedonic quality words include ungenerous, tacky, irksome and so on. These results can be used to enlighten designers for exploring optimisation on battery. Therefore, hedonic quality analysis is capable of providing sights for designers to understand the product defects.

There are some insights derived from experiments. Firstly, the proposed data collection method is superior to the traditional collection methods in hedonic quality studies. The traditional

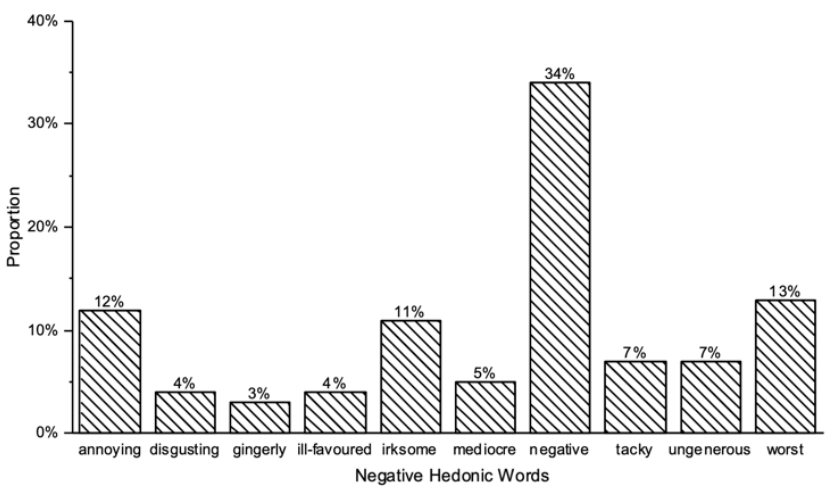

FIGURE 9. NEGATIVE HEDONIC WORDS IN “BATTERY”

hedonic quality questionnaire is much more time-consuming.
Furthermore, this method improves the scope of data collection, which largely enriches the range of customer reviews. Secondly, extracting hedonic quality factors from online customer reviews can provide in-depth reflections for designers to improve their product design. Finally, integrating hedonic quality for UX modelling enriches the online review UX measurement method.

\section{CONCLUSIONS}

In the past few years, there is an increasing trend that researchers focus on UX analysis. Based on user-centric design, human factors, and the advantages of ergonomics, design researchers have recognised the benefits of UX. A delighted UX from customers can lead to the success of a product or service in markets.

\subsection{Theoretical contributions}

There are some significant theoretical contributions made by this study. First of all, this study introduces a UX modelling approach by integrating hedonic quality based on hybrid machine learning algorithms. The data used in the study were collected from online popular social networking and ecommerce site that is generated by a large number of users (e.g., product reviews and consumers opinion). The proposed method provides a scientific and automatic way to collect and manage hedonic quality information from online reviews. Compared with the traditional hedonic quality measurement methods, our approach is more efficient and can be further used to measure the hedonic quality in the UX research area.

\subsection{Implication for practice}

In the case study, the smartphone comments on how to extract the UX data and provide critical product features based on UX integration results and an excellent example of using the context. Therefore, each particular individual user put forward the UX microcosmic view and provides the UX macro view by different user collective experience part like hedonic part. Based on this research, the hedonic quality will influence customer experience when interacting with a product or service. For example, providing users with similar product characteristics and function in different contexts could create an entirely different hedonic quality, such as different levels of emotion, surprisingly pleasant or disappointing feelings.

This study provides essential enlightenment for strategic design and tactical activities in design activities. It can be used to assist the design concept generation and UX design. Additionally, this study can provide insights for predictive UX. The introduced approach can help designers or researchers to tackle the challenges in existing UX analysis and modelling. Conventional surveys and questionnaires are heavily counted on labour, so the information related to UX, user profiles, and contexts are inevitably limited, not to mention that it is timeconsuming for collecting such data.

\subsection{Limitations and opportunities}

On the technical level, further improvement can be made to enhance the discovery of UX, analysis and reasoning of UX, and 
construction of the corresponding knowledge base. One possible route is to append an incremental learning process that extends the existing domain knowledge base. Additionally, although Amazon.com is the world's largest e-commercial website and provides enormous product reviews, our results can be further enriched if more relevant reviews from other sites, e.g., eBay or shopping.com, are considered. Simultaneously, the hedonic quality analysis can be applied throughout the entire product lifecycle from the early stage (e.g., advertising) to final interaction with customers. This will be considered in our further study to better evaluate hedonic quality for products and services.

On evaluating the proposed UX modelling and the impact of predictive UX, virtual reality (VR) technologies (e.g., digital games) become increasingly important to expand the evaluation of using context and UX designed [37]. This can be used for capturing hedonic qualities from users more precisely and directly. Before the products are launched to markets, the VR game's tests are promising to be used for products evaluation, where insights for further improvement of design can be derived.

\section{REFERENCES}

[1] Pucillo, F., and Cascini, G., 2014, "A framework for user experience, needs and affordances," Design Studies, 35(2), pp. 160-179.

[2] Lin, C. J., and Cheng, L.-Y., 2017, "Product attributes and user experience design: how to convey product information through user-centered service," Journal of Intelligent Manufacturing, 28(7), pp. 1743-1754.

[3] Hassenzahl, M., "User experience (UX) towards an experiential perspective on product quality," Proc. Proceedings of the 20th Conference on l'Interaction Homme-Machine, pp. 1115.

[4] Law, E. L.-C., Roto, V., Hassenzahl, M., Vermeeren, A. P., and Kort, J., "Understanding, scoping and defining user experience: a survey approach," Proc. Proceedings of the SIGCHI conference on human factors in computing systems, pp. 719-728.

[5] ISO, B., and STANDARD, B., 2010, "Ergonomics of human-system interaction."

[6] Hassenzahl, M., 2005, "The thing and I: understanding the relationship between user and product," Funology: from usability to enjoyment, pp. 31-42.

[7] Chen, Z., Ma, N., and Liu, B., 2018, "Lifelong learning for sentiment classification," arXiv preprint arXiv:1801.02808.

[8] Ahmad, S. N., and Laroche, M., 2017, "Analysing electronic word of mouth: A social commerce construct," International Journal of Information Management, 37(3), pp. 202-213.

[9] Zhan, J., Loh, H. T., and Liu, Y., 2009, "Gather customer concerns from online product reviews-A text summarisation approach," Expert Systems with Applications, 36(2), pp. 21072115.
[10] He, W., 2013, "Improving user experience with casebased reasoning systems using text mining and Web 2.0," Expert Systems with Applications, 40(2), pp. 500-507.

[11] Yang, B., Liu, Y., Liang, Y., and Tang, M., 2019, "Exploiting user experience from online customer reviews for product design," International Journal of Information Management, 46, pp. 173-186.

[12] Hassenzahl, M., and Tractinsky, N., 2006, "User experience-a research agenda," Behaviour \& information technology, 25(2), pp. 91-97.

[13] Mahlke, S., and Thüring, M., "Studying antecedents of emotional experiences in interactive contexts," Proc. Proceedings of the SIGCHI conference on Human factors in computing systems, pp. 915-918.

[14] Brandenburg, S., Vogel, M., and Drewitz, U., "User experience starts at the keystroke level: The Model of User Experience (MUX)," Proc. International Conference of Design, User Experience, and Usability, Springer, pp. 449-458.

[15] Merčun, T., and Žumer, M., 2017, "Exploring the influences on pragmatic and hedonic aspects of user experience."

[16] Minge, M., "Dynamics of user experience," Proc. Proceedings of the Workshop on Research Goals and Strategies for Studying User Experience and Emotion, NordiCHI.

[17] Van der Heijden, H., 2004, "User acceptance of hedonic information systems," MIS quarterly, pp. 695-704.

[18] Hassenzahl, M., Platz, A., Burmester, M., and Lehner, K., "Hedonic and ergonomic quality aspects determine a software's appeal," Proc. Proceedings of the SIGCHI conference on Human factors in computing systems, pp. 201-208.

[19] Hassenzahl, M., 2001, "The effect of perceived hedonic quality on product appealingness," International Journal of Human-Computer Interaction, 13(4), pp. 481-499.

[20] Laugwitz, B., Held, T., and Schrepp, M., "Construction and evaluation of a user experience questionnaire," Proc. Symposium of the Austrian HCI and usability engineering group, Springer, pp. 63-76.

[21] Minge, M., Riedel, L., and Thüring, M., 2014, "Modulare Evaluation interaktiver Technik. Entwicklung und Validierung des meCUE Fragebogens zur Messung der User Experience," Grundlagen und Anwendungen der MenschTechnik-Interaktion, 10, pp. 28-36.

[22] Hazlett, R. L., and Benedek, J., 2007, "Measuring emotional valence to understand the user's experience of software," International Journal of Human-Computer Studies, 65(4), pp. 306-314.

[23] Hassenzahl, M., Diefenbach, S., and Göritz, A., 2010, "Needs, affect, and interactive products-Facets of user experience," Interacting with computers, 22(5), pp. 353-362.

[24] Kujala, S., Roto, V., Väänänen-Vainio-Mattila, K., Karapanos, E., and Sinnelä, A., 2011, "UX Curve: A method for evaluating long-term user experience," Interacting with computers, 23(5), pp. 473-483.

[25] Jin, J., Liu, Y., Ji, P., and Kwong, C., 2019, "Review on recent advances in information mining from big consumer opinion data for product design," Journal of Computing and Information Science in Engineering, 19(1). 
[26] Jin, J., and Liu, Y., "How to interpret the helpfulness of online product reviews: bridging the needs between customers and designers," Proc. Proceedings of the 2nd international workshop on Search and mining user-generated contents, pp. 8794.

[27] Dave, K., Lawrence, S., and Pennock, D. M., "Mining the peanut gallery: Opinion extraction and semantic classification of product reviews," Proc. Proceedings of the 12th international conference on World Wide Web, pp. 519-528.

[28] Ding, X., Liu, B., and Zhang, L., "Entity discovery and assignment for opinion mining applications," Proc. Proceedings of the 15th ACM SIGKDD international conference on Knowledge discovery and data mining, pp. 1125-1134.

[29] Wang, H., Lu, Y., and Zhai, C., "Latent aspect rating analysis on review text data: a rating regression approach," Proc. Proceedings of the 16th ACM SIGKDD international conference on Knowledge discovery and data mining, pp. 783-792.

[30] Popescu, A.-M., and Etzioni, O., 2007, "Extracting product features and opinions from reviews," Natural language processing and text mining, Springer, pp. 9-28.

[31] Brill, E., and Marcus, M., 1992, "Automatically acquiring phrase structure using distributional analysis," PENNSYLVANIA UNIV PHILADELPHIA.

[32] Losee, R. M., 2001, "Natural language processing in support of decision-making: phrases and part-of-speech tagging," Information processing \& management, 37(6), pp. 769-787.

[33] Pelleg, D., and Moore, A. W., "X-means: Extending kmeans with efficient estimation of the number of clusters," Proc. Icml, pp. 727-734.

[34] Liu, Y., and Loh, H. T., "Corpus building for corporate knowledge discovery and management: A case study of manufacturing," Proc. International Conference on KnowledgeBased and Intelligent Information and Engineering Systems, Springer, pp. 542-550.

[35] Manning, C., and Schutze, H., 1999, Foundations of statistical natural language processing, MIT press.

[36] Jones, K. S., and Willett, P., 1997, Readings in information retrieval, Morgan Kaufmann.

[37] Engl, S., and Nacke, L. E., 2013, "Contextual influences on mobile player experience-A game user experience model," Entertainment Computing, 4(1), pp. 83-91. 